\title{
Further results on the generalized Mittag-Leffler function operator
}

\author{
Ram K Saxena ${ }^{1}$, Jignesh P Chauhan², Ranjan K Jana² and Ajay K Shukla²*
}

${ }^{\text {*Correspondence: }}$ ajayshukla2@rediffmail.com

2Department of Applied Mathematics \& Humanities,

S.V. National Institute of Technology, Surat, 395007, India

Full list of author information is available at the end of the article

\begin{abstract}
The present paper deals with the study of a generalized Mittag-Leffler function operator. This paper is based on the generalized Mittag-Leffler function introduced and studied by Saxena and Nishimoto (J. Fract. Calc. 37:43-52, 2010). Laplace and Mellin transforms of this new operator are investigated. The results are useful where the MittagLeffler function occurs naturally. The boundedness and composition properties of this operator are established. The importance of the derived results further lies in the fact that the results of the generalized Mittag-Leffler function defined by Prabhakar (Yokohama Math. J. 19:7-15, 1971), Shukla and Prajapati (J. Math. Anal. Appl. 336:797-811, 2007), and the multiindex Mittag-Leffler function due to Kiryakova (Fract. Calc. Appl. Anal. 2:445-462, 1999; J. Comput. Appl. Math. 118:214-259, 2000; J. Fract. Calc. 40:29-41, 2011) readily follow as a special case of our findings. Further the results obtained are of general nature and include the results given earlier by Prajapati et al. (J. Inequal. Appl. 2013:33, 2013) and Srivastava and Tomovski (Appl. Math. Comput. 211:198-210, 2009). Some special cases of the established results are also given as corollaries.
\end{abstract}

MSC: 33E12; 44A10; 26A33

Keywords: generalized Mittag-Leffler function; Laplace transform; Mellin transform; $H$-function; Mellin-Barnes type integrals; Riemann-Liouville fractional integral; Hilfer derivative

\section{Introduction}

The Swedish mathematician Gösta Mittag-Leffler in the year 1903 introduced the function $[1,2]$

$$
E_{\alpha}(z)=\sum_{n=0}^{\infty} \frac{z^{n}}{\Gamma(\alpha n+1)} \quad(\alpha \in C ; \operatorname{Re}(\alpha)>0) .
$$

In the year 1905, Wiman [3] introduced a generalization of (1) in the form

$$
E_{\alpha, \beta}(z)=\sum_{n=0}^{\infty} \frac{z^{n}}{\Gamma(\alpha n+\beta)} \quad(\alpha, \beta \in C ; \operatorname{Re}(\alpha)>0, \operatorname{Re}(\beta)>0) .
$$

In connection with the solution of an integral equation, Prabhakar [4] in the year 1971 introduced a very interesting useful generalization of (2) in the form

$$
E_{\alpha, \beta}^{\gamma}(z)=\sum_{n=0}^{\infty} \frac{(\gamma)_{n} z^{n}}{\Gamma(\alpha n+\beta)}
$$


where $\alpha, \beta, \gamma \in C, \operatorname{Re}(\alpha)>0, \operatorname{Re}(\beta)>0,(\gamma)_{0}=1$, and

$$
(\gamma)_{n}=\gamma(\gamma+1) \cdots(\gamma+n-1)=\frac{\Gamma(\gamma+n)}{\Gamma(\gamma)} ; \quad \gamma \neq 0 .
$$

In the year 2007, Shukla and Prajapati [5] introduced the following generalization of (3):

$$
E_{\alpha, \beta}^{\gamma, q}(z)=\sum_{n=0}^{\infty} \frac{(\gamma)_{q n} z^{n}}{\Gamma(\alpha n+\beta)}
$$

where $\alpha, \beta, \gamma \in C ; \operatorname{Re}(\alpha)>0, \operatorname{Re}(\beta)>0(q \in(0,1) \cup \mathrm{N})$.

The above generalization is studied by Shukla and Prajapati [5], Prajapati et al. [6] and established relation with Wright function [7-10]. In the year 2009, a generalization of (5) was introduced by Srivastava and Tomovski [11],

$$
E_{\alpha, \beta}^{\gamma, \kappa}(z)=\sum_{n=0}^{\infty} \frac{(\gamma)_{\kappa n} z^{n}}{\Gamma(\alpha n+\beta)}
$$

where $\alpha, \beta, \gamma \in C ; \operatorname{Re}(\alpha)>\max [0, \operatorname{Re}(\kappa)-1], \min \{\operatorname{Re}(\beta), \operatorname{Re}(\kappa)\}>0$.

The Pochhammer symbol is defined by

$$
(\lambda)_{\mu}=\frac{\Gamma(\lambda+\mu)}{\Gamma(\lambda)}= \begin{cases}1, & \mu=0, \lambda, \mu \in C \backslash(0) \\ \lambda(\lambda+1) \cdots(\lambda+n-1), & \mu=n \in \mathrm{N} ; \lambda \in C\end{cases}
$$

A further generalization of the Mittag-Leffler function defined by Shukla and Prajapati [5] was given by Saxena et al. [12] in the year 2010 as

$$
E_{\gamma, q}\left[\left(\alpha_{1}, \beta_{1}\right), \ldots,\left(\alpha_{m}, \beta_{m}\right) ; z\right]=\sum_{n=0}^{\infty} \frac{(\gamma)_{q n} z^{n}}{\prod_{j=1}^{m} \Gamma\left(n \alpha_{j}+\beta_{j}\right)(n) !},
$$

where $\alpha_{j}, \beta_{j}, \gamma \in C, \operatorname{Re}\left(\alpha_{j}\right)>0, \operatorname{Re}\left(\beta_{j}\right)>0(j=1, \ldots, m), \operatorname{Re}\left(\sum_{j=1}^{m} \alpha_{j}\right)>\max [0, q-1](q \in$ $(0,1) \cup N)$.

This was further generalized by Saxena and Nishimoto $[13,14]$ in the year 2010 in the following form:

$$
E_{\gamma, \kappa}\left[\left(\alpha_{1}, \beta_{1}\right), \ldots,\left(\alpha_{m}, \beta_{m}\right) z\right]=\sum_{n=0}^{\infty} \frac{(\gamma)_{\kappa n} z^{n}}{\prod_{j=1}^{m} \Gamma\left(n \alpha_{j}+\beta_{j}\right)(n) !},
$$

where $\alpha_{j}, \beta_{j}, \gamma, \kappa \in C, \operatorname{Re}\left(\alpha_{j}\right)>0, \operatorname{Re}\left(\beta_{j}\right)>0(j=1, \ldots, m) ; \operatorname{Re}(\kappa)>0, \operatorname{Re}\left(\sum_{j=1}^{m} \alpha_{j}\right)>$ $\max [0, \kappa-1]$.

If we set $\gamma=\kappa=1$ in (9), it reduces to the following multiindex Mittag-Leffler function studied by Kiryakova [15-17]:

$$
E_{1,1}\left[\left(\alpha_{1}, \beta_{1}\right), \ldots,\left(\alpha_{m}, \beta_{m}\right) z\right]=\sum_{n=0}^{\infty} \frac{z^{n}}{\prod_{j=1}^{m} \Gamma\left(n \alpha_{j}+\beta_{j}\right)},
$$

where $\alpha_{j}, \beta_{j} \in C, \operatorname{Re}\left(\alpha_{j}\right)>0, \operatorname{Re}\left(\beta_{j}\right)>0(j=1, \ldots, m)$.

For $\kappa=q(q \in(0,1) \cup \mathrm{N}),(9)$ reduces to the one studied by Saxena et al. [12].

The following lemma was proved by Saxena and Nishimoto [14]. 
Lemma [18] Let $\alpha_{j}, \beta_{j}, \gamma, \kappa \in C, \operatorname{Re}\left(\alpha_{j}\right)>0, \operatorname{Re}\left(\beta_{j}\right)>0(j=1, \ldots, m) ; \operatorname{Re}(\kappa)>0, \operatorname{Re}\left(\sum_{j=1}^{m} \alpha_{j}\right)>$ $\max [0, \kappa-1]$, then the function defined by (10) is represented by the Mellin-Barnes type integral as follows:

$$
E_{\gamma, \kappa}\left[\left(\alpha_{1}, \beta_{1}\right), \ldots,\left(\alpha_{m}, \beta_{m}\right) ; z\right]=\frac{1}{2 \pi i \Gamma(\gamma)} \int_{-i \infty}^{+i \infty} \frac{\Gamma(\xi) \Gamma(\gamma-\kappa \xi)(-z)^{-\xi} d \xi}{\prod_{j=1}^{m} \Gamma\left(\beta_{j}-\xi \alpha_{j}\right)}
$$

where the contour of integration starts at $-i \infty$ and ends at $+i \infty$ and separates the poles at the point $\xi=-n\left(n \in \mathrm{N}_{0}\right)$ to the left and all the poles of $\Gamma(\gamma-\kappa \xi)$ at the point $\xi=\frac{\gamma}{\kappa}+n$ $\left(n \in \mathrm{N}_{0}\right)$ to the right.

The Riemann-Liouville fractional integral operator of $f(t)$ is defined as [14, 19-22]

$$
\left(I_{a+1}^{\alpha} f\right)(x)=\frac{1}{\Gamma(\alpha)} \int_{a}^{x}(x-t)^{\alpha-1} f(t) d t \quad(\alpha \in C ; \operatorname{Re}(\alpha)>0) .
$$

The Riemann-Liouville fractional derivative operator of $f(t)$ is defined by $[14,19-23]$

$$
\left(D_{a+}^{\mu} f\right)(x)= \begin{cases}\frac{1}{\Gamma(-\mu)} \int_{a}^{x}(x-t)^{-\mu-1} f(t) d t & (\operatorname{Re}(\mu)<0), \\ \frac{d^{m}}{d x^{m}} D_{a+}^{\mu-m} f(x) & (m-1 \leq \operatorname{Re}(\mu)<m)(m \in \mathrm{N}) .\end{cases}
$$

The Caputo fractional derivative was defined by Caputo [24] in the form

$$
\begin{aligned}
{ }_{0}^{C} D_{t}^{\alpha} f(t) & =\frac{\partial^{m} f(t)}{\partial t^{m}} \frac{1}{\Gamma(m-\alpha)} \int_{0}^{t} \frac{f^{m}(\tau) d \tau}{(t-\tau)^{\alpha+1-m}} \quad(m-1<\alpha<m ; \operatorname{Re}(\alpha)>0, m \in \mathrm{N}) \\
& =\frac{\partial^{m} f(t)}{\partial t^{m}} \quad \text { if } \alpha=m,
\end{aligned}
$$

where $\frac{\partial^{m} f(t)}{\partial t^{m}}$ is the $m$ th partial derivative of $f(t)$ with respect to $t$.

The Fox's $H$-function $[20,25]$ is defined in terms of the Mellin-Barnes type integral in the form

$$
\begin{aligned}
H_{p, q}^{m, n}(z) & =H_{p, q}^{m, n}\left[z \mid \begin{array}{c}
\left(b_{q}, B_{q}\right) \\
\left(a_{p}, A_{p}\right)
\end{array}\right]=H_{p, q}^{m, n}\left[z \mid \begin{array}{c}
\left(b_{1}, B_{1}\right), \ldots,\left(b_{q}, B_{q}\right) \\
\left(a_{1}, A_{1}\right), \ldots,\left(a_{p}, A_{p}\right)
\end{array}\right] \\
& =\frac{1}{2 \pi i} \int_{\Omega} \Theta(\xi) z^{-\xi} d \xi,
\end{aligned}
$$

where

$$
\Theta(\xi)=\frac{\left[\prod_{i=1}^{m} \Gamma\left(b_{i}+B_{i} \xi\right)\right]\left[\prod_{j=1}^{n} \Gamma\left(1-a_{j}-A_{j} \xi\right)\right]}{\left[\prod_{i=m+1}^{q} \Gamma\left(1-b_{i}-B_{i} \xi\right)\right]\left[\prod_{j=n+1}^{p} \Gamma\left(a_{j}+A_{j} \xi\right)\right]} .
$$

A detailed definition, properties, asymptotic expansion and a comprehensive account of the $H$-function is available from the monographs written by Mathai et al. [20].

The generalized Wright function $\Psi_{q}\left(p, q \in \mathrm{N}_{0}\right)$ is defined as ([26], p.183)

$$
{ }_{p} \Psi_{q}\left[\begin{array}{c}
\left(a_{1}, A_{1}\right), \ldots,\left(a_{p}, A_{p}\right) ; z \\
\left(b_{1}, B_{1}\right), \ldots,\left(b_{q}, B_{q}\right) ;
\end{array}\right]=\sum_{n=0}^{\infty} \prod_{n=0}^{\infty} \frac{\Gamma\left(a_{1}+n A_{1}\right) \cdots \Gamma\left(a_{p}+n A_{p}\right)}{\Gamma\left(b_{1}+n B_{1}\right) \cdots \Gamma\left(b_{q}+n B_{q}\right)} \frac{z^{n}}{n !},
$$




$$
\begin{aligned}
{ }_{p} \Psi_{q} *\left[\begin{array}{l}
\left(a_{1}, A_{1}\right), \ldots,\left(a_{p}, A_{p}\right) ; z \\
\left(b_{1}, B_{1}\right), \ldots,\left(b_{q}, B_{q}\right) ;
\end{array}\right] & =\sum_{n=0}^{\infty} \frac{\left(a_{1}\right)_{A_{1} n} \cdots\left(a_{p}\right)_{A_{p} n}}{(b)_{B_{1} n} \cdots\left(b_{q}\right)_{B_{q} n}} \frac{z^{n}}{n !} \\
& =\frac{\Gamma\left(b_{1}\right) \cdots \Gamma\left(b_{q}\right)}{\Gamma\left(a_{1}\right) \cdots \Gamma\left(a_{p}\right)} p \Psi_{q}\left[\begin{array}{l}
\left(a_{1}, A_{1}\right), \ldots,\left(a_{p}, A_{p}\right) ; z \\
\left(b_{1}, B_{1}\right), \ldots,\left(b_{q}, B_{q}\right) ;
\end{array}\right]
\end{aligned}
$$

where

$$
A_{j} \in R(j=1, \ldots, p), B_{j} \in R(j=1, \ldots, q) ; \quad 1+\sum_{j=0}^{q} B_{j}-\sum_{j=0}^{p} A_{j} \geq 0,
$$

and the equality in the above condition holds true for suitably bounded values of $|z|$ given by $|z|<\nabla:=\left(\prod_{j=1}^{p} A_{j}{ }^{-A_{j}}\right)\left(\prod_{j=1}^{q} B_{j}^{B_{j}}\right)$.

In particular, when $A_{j}=B_{k}=1(j=1, \ldots, p$ and $k=1, \ldots, q)$, the following relationship holds true:

$$
\begin{aligned}
{ }_{p} \Psi_{q} *\left[\begin{array}{l}
\left(a_{1}, 1\right), \ldots,\left(a_{p}, 1\right) ; \\
\left(b_{1}, 1\right), \ldots,\left(b_{q}, 1\right) ;
\end{array}\right] & ={ }_{p} F_{q}\left[\begin{array}{l}
a_{1}, \ldots, a_{p} ; \\
b_{1}, \ldots, b_{q} ;
\end{array}\right]=\sum_{n=0}^{\infty} \frac{\left(a_{1}\right)_{n} \cdots\left(a_{p}\right)_{n}}{(b)_{n} \cdots\left(b_{q}\right)_{n}} \frac{z^{n}}{n !} \\
& =\frac{\Gamma\left(b_{1}\right) \ldots \Gamma\left(b_{q}\right)}{\Gamma\left(a_{1}\right) \cdots \Gamma\left(a_{p}\right)} p \Psi_{q}\left[\begin{array}{l}
\left(a_{1}, 1\right), \ldots,\left(a_{p}, 1\right) ; \\
\left(b_{1}, 1\right), \ldots,\left(b_{q}, 1\right) ;
\end{array}\right]
\end{aligned}
$$

in terms of the generalized hypergeometric function ${ }_{p} F_{q}\left(p, q \in \mathrm{N}_{0}\right)$.

Recently, Górska et al. [27] studied operator solution for fractional Fokkar-Planck equations and Babusci et al. [28] investigated Mittag-leffler function and associated polynomials.

A generalization of Riemann-Liouville fractional derivative operator (13) as well as Caputo fractional derivative operator (14) was given by Hilfer [18] by introducing a rightsided fractional derivative operator of two parameters of order $0<\mu<1$ and $0 \leq v \leq 1$ in the form

$$
D_{a+}^{\mu, v} N(x, t)=\left(I_{a+}^{v(1-\mu)} \frac{\partial}{\partial x}\left(I_{a+}^{(1-v)(1-\mu)} N(x, t)\right)\right)
$$

where the initial value term $\left(I_{0+}^{(1-\nu)(1-\mu)} f\right)(0+)$ involves the Riemann-Liouville fractional integral of order $(1-\nu)(1-\mu)$ evaluated in the limit as $t \rightarrow 0+$ provided that the Laplace transform exists.

The object of this paper is to derive certain properties including the Laplace transform and the Mellin transform of the integral operator associated with the generalized MittagLeffler function defined by

$$
\left(E_{\gamma, \kappa ; \beta_{1}, \ldots, \beta_{m} ; \omega, a+}^{\alpha_{1}, \ldots, \alpha_{m}} \varphi\right)(x)=\int_{a}^{x}(x-t)^{\beta_{1}-1} E_{\gamma, \kappa ; \beta_{1}, \ldots, \beta_{m}}^{\alpha_{1}, \ldots, \alpha_{m}}\left[\omega(x-t)^{\alpha_{1}}\right] \varphi(t) d t \quad(x>a)
$$

where $\alpha_{j}, \beta_{j}, \gamma, \kappa, \omega \in C, \operatorname{Re}\left(\alpha_{j}\right)>0, \operatorname{Re}\left(\beta_{j}\right)>0(j=1, \ldots, m) ; \operatorname{Re}\left(\sum_{j=1}^{m} \alpha_{j}\right)>\max [0, \kappa-1]$, $\operatorname{Re}(\gamma)>0, \operatorname{Re}(\kappa)>0$.

This operator includes, as special cases, the operators defined and studied by Kilbas et al. [29], Shukla and Prajapati [5], Srivastava and Tomovski [11] and Kiryakova [16]. If 
$\kappa=m=1$ in (22) we obtain the following fractional integration operator defined by Kilbas et al. [29]:

$$
\left(E_{\gamma, \beta ; \omega, a+}^{\alpha} \varphi\right)(x)=\int_{a}^{x}(x-t)^{\beta-1} E_{\gamma, \beta}^{\alpha}\left[\omega(x-t)^{\alpha}\right] \varphi(t) d t \quad(x>a),
$$

where $\alpha, \beta, \gamma, \omega \in C ; \operatorname{Re}(\alpha)>0, \operatorname{Re}(\beta)>0$.

As $\gamma$ tends to zero, then by virtue of the limit formula

$$
E_{\gamma, \beta}^{\alpha}(z)=\frac{1}{\Gamma(\beta)},
$$

equation (22) reduces to the familiar Riemann-Liouville fractional integral defined by (12).

If we set $m=1$ in (22), it gives the integral operator defined by Srivastava and Tomovski [11]

$$
\left(E_{\gamma, \kappa, \beta ; \omega, a+}^{\alpha} \varphi\right)(x)=\int_{a}^{x}(x-t)^{\beta_{1}-1} E_{\alpha, \beta}^{\gamma, \kappa}\left[\omega(x-t)^{\alpha}\right] \varphi(t) d t \quad(x>a)
$$

where $\alpha, \beta, \gamma, \omega \in C ; \operatorname{Re}(\alpha)>0, \operatorname{Re}(\beta)>0, \operatorname{Re}(\kappa)>0, \operatorname{Re}(\alpha)=\operatorname{Re}(\kappa)-1>0$, which for $\kappa=q$ reduces to the one given by Shukla and Prajapati [5].

If, however, we take $\gamma=\kappa=1$, (22) yields the following integral operator associated with the multiindex Mittag-Leffler function defined by Kiryakova [16]:

$$
\left(E_{\gamma, \kappa ; \beta_{1}, \ldots, \beta_{m} ; \omega, a+}^{\alpha_{1}, \ldots, \alpha_{m}} \varphi\right)(x)=\int_{0}^{x}(x-t)^{\beta_{1}-1} E_{\gamma, \kappa ; \beta_{1}, \ldots, \beta_{m}}^{\alpha_{1}, \ldots, \alpha_{m}}\left[\omega(x-t)^{\alpha_{1}}\right] \varphi(t) d t \quad(x>a),
$$

where $\alpha_{j}, \beta_{j}, \omega \in C, \operatorname{Re}\left(\alpha_{j}\right)>0, \operatorname{Re}\left(\beta_{j}\right)>0(j=1, \ldots, m)$.

We now proceed to derive the Mellin transform of the integral operator defined by (22).

\section{Mellin transform of the integral operator defined by (22)}

Theorem 2.1 It will be shown here that

$$
\begin{aligned}
& M\left[\left(E_{\gamma, \ldots ; \beta_{1}, \ldots, \beta_{m} ; \omega, a+}^{\alpha_{1} \ldots, \alpha_{m}} \varphi\right)(x) ; s\right] \\
& =\frac{1}{\Gamma(\gamma) \Gamma(1-s)} H_{1, m+1}^{2,1}\left[-\omega t^{\alpha} \mid \begin{array}{c}
(1-\gamma, \kappa) \\
(0,1),\left(1-s-\beta_{1}, \alpha_{1}\right),\left(1-s-\beta_{j}, \alpha_{j}\right)_{2, m}
\end{array}\right] \\
& \quad \times M\left[t^{\beta_{1}} \varphi(t)\right],
\end{aligned}
$$

where $\alpha_{j}, \beta_{j}, \gamma, \kappa, \omega \in C, \operatorname{Re}\left(\alpha_{j}\right)>0, \operatorname{Re}\left(\beta_{j}\right)>0(j=1, \ldots, m) ; \operatorname{Re}\left(\sum_{j=1}^{m} \alpha_{j}\right)>\max [0, \kappa-1]$, $\operatorname{Re}(\gamma)>0, \operatorname{Re}(\kappa)>0, \operatorname{Re}\left(1-s-\beta_{1}\right)>0$ and $H_{1, m+1}^{2,1}(\cdot)$ is the H-function defined by (15).

Proof The Mellin transform of a function $f(t)$ is defined by

$$
M[\varphi(x) ; s]=\int_{0}^{\infty} x^{s-1} \varphi(x) d x .
$$

Therefore we have

$$
M\left[\left(E_{\gamma, \kappa ; \beta_{1}, \ldots, \beta_{m} ; \omega, a+}^{\alpha_{1}, \ldots, \alpha_{m}} \varphi\right)(x) ; s\right]=\int_{0}^{\infty} x^{s-1} \int_{0}^{x}(x-t)^{\beta_{1}-1} E_{\gamma, \kappa ; \beta_{1}, \ldots, \beta_{m}}^{\alpha_{1}, \ldots, \alpha_{m}}\left[\omega(x-t)^{\alpha_{1}}\right] \varphi(t) d t d x
$$


where $x>0$ and interchanging the order of integration, which is permissible under the conditions stated in Theorem 2.1, we find that

$$
\begin{aligned}
M & {\left[\left(E_{\gamma, k ; \beta_{1}, \ldots, \beta_{m} ; \omega, a+}^{\alpha_{1}, \ldots, \alpha_{m}} \varphi\right)(x) ; s\right] } \\
& =\int_{0}^{\infty} \varphi(t) \int_{t}^{\infty} x^{s-1}(x-t)^{\beta_{1}-1} E_{\gamma, \kappa ; \beta_{1}, \ldots, \beta_{m}}^{\alpha_{1}, \ldots, \alpha_{m}}\left[\omega(x-t)^{\alpha_{1}}\right] d x d t .
\end{aligned}
$$

If we set $x=t+u$ on the right-hand side of (29), it gives

$$
M\left[\left(E_{\gamma, \kappa ; \beta_{1}, \ldots, \beta_{m} ; \omega, a+}^{\alpha_{1} \ldots, \alpha_{m}} \varphi\right)(x) ; s\right]=\int_{0}^{\infty} \varphi(t) \int_{0}^{\infty}(t+u)^{s-1} u^{\beta_{1}-1} E_{\gamma, \kappa ; \beta_{1}, \ldots, \beta_{m}}^{\alpha_{1}, \ldots, \alpha_{m}}\left[\omega u^{\alpha_{1}}\right] d u d t
$$

To evaluate the $u$-integral, we express the generalized Mittag-Leffler function in terms of its Mellin-Barnes contour integral by means of formula (11), then the above integral transforms into the form

$$
\begin{aligned}
& M\left[\left(E_{\gamma, \kappa ; \beta_{1}, \ldots, \beta_{m} ; \omega, a+}^{\alpha_{1}, \ldots, \alpha_{m}} \varphi\right)(x) ; s\right] \\
& =\int_{0}^{\infty} \varphi(t) d t \frac{1}{2 \pi i \Gamma(\gamma)} \int_{-i \infty}^{+i \infty} \frac{\Gamma(-\xi) \Gamma(\gamma+\kappa \xi)\left(-\omega u^{\alpha}\right)^{\xi} d \xi}{\prod_{j=1}^{m} \Gamma\left(\beta_{j}-\xi \alpha_{j}\right)} \\
& \quad \times \int_{0}^{\infty}(t+u)^{s-1} u^{\beta_{1}-1} d u .
\end{aligned}
$$

Now we evaluate the $u$-integral with the help of the formula [30]

$$
\int_{0}^{\infty} t^{\nu-1}(t+a)^{-\rho} d t=\frac{\Gamma(v) \Gamma(\rho-v)}{\Gamma(\rho)} a^{\nu-\rho}
$$

where $\operatorname{Re}(v)>0, \operatorname{Re}(\rho-v)>|\arg (-a)|<\pi$; we observe that the right-hand side of the above equation (32) and the definition of $H$-function (15) yields the desired result (27).

Corollary 2.1 For $\kappa=q$, Theorem 2.1 reduces to the following result:

$$
\begin{aligned}
& M\left[\left(E_{\gamma, q ; \beta_{1}, \ldots, \beta_{m} ; \omega, a+\varphi}^{\alpha_{1}, \ldots, \alpha_{m}}\right)(x) ; s\right] \\
& =\frac{1}{\Gamma(\gamma) \Gamma(1-s)} \times H_{1, m+1}^{2,1}\left[-\omega t^{\alpha} \mid \begin{array}{c}
(1-\gamma, q) \\
(0,1),\left(1-s-\beta_{1}, \alpha_{1}\right),\left(1-s-\beta_{j}, \alpha_{j}\right)_{2, m}
\end{array}\right] \\
& \quad \times M\left[t^{\beta_{1}} \varphi(t)\right],
\end{aligned}
$$

which holds under the conditions as given with (27) with $\kappa=q(q \in(0,1) \cup \mathrm{N})$.

When $m=1$, Corollary 2.1 reduces to the following one given by Prajapati et al. [6].

Corollary 2.2 There holds the following result:

$$
\begin{aligned}
& M\left[\left(E_{\gamma, q ; \beta ; \omega, 0+}^{\alpha} \varphi\right)(x) ; s\right] \\
& \quad=\frac{1}{\Gamma(\gamma) \Gamma(1-s)} \times H_{1, m+1}^{2,1}\left[\begin{array}{l|c}
-\omega t^{\alpha} & (1-\gamma, q) \\
(0,1),(1-s-\beta, \alpha)
\end{array}\right] M\left[t^{\beta} \varphi(t)\right] .
\end{aligned}
$$


If we further take $q=1$, Corollary 2.2 reduces to the following result [6].

Corollary 2.3 There holds the following result:

$$
\begin{aligned}
M & {\left[\left(E_{\gamma ; \beta ; \omega, 0+}^{\alpha} \varphi\right)(x) ; s\right] } \\
& =\frac{1}{\Gamma(\gamma) \Gamma(1-s)} \times H_{1,2}^{2,1}\left[\begin{array}{l|c}
-\omega t^{\alpha} & (1-\gamma, 1) \\
(0,1),(1-s-\beta, \alpha)
\end{array}\right] M\left[t^{\beta} \varphi(t)\right] .
\end{aligned}
$$

For $m=1$, Theorem 2.1 gives the following result given in [6].

Corollary 2.4 There holds the formula

$$
\begin{aligned}
& M\left[\left(E_{\gamma, \kappa ; \beta ; \omega, 0+}^{\alpha} \varphi\right)(x) ; s\right] \\
& \quad=\frac{1}{\Gamma(\gamma) \Gamma(1-s)} \times H_{1,2}^{2,1}\left[\begin{array}{l|c}
-\omega t^{\alpha} & (1-\gamma, \kappa) \\
(0,1),(1-s-\beta, \alpha)
\end{array}\right] M\left[t^{\beta} \varphi(t)\right],
\end{aligned}
$$

where $\alpha, \beta, \gamma \in C ; \operatorname{Re}(\alpha)>\max [0, \operatorname{Re}(\kappa)-1], \operatorname{Re}(\beta)>0, \operatorname{Re}(\kappa)>0$.

When $\kappa=1$, it yields the following result associated with the multiindex Mittag-Leffler function $[15,16]$.

Corollary 2.5 The following result holds:

$$
\begin{aligned}
M & {\left[\left(E_{\beta_{1}, \ldots, \beta_{m} ; \omega, 0+}^{\alpha_{1}, \ldots, \alpha_{m}} \varphi\right)(x) ; s\right] } \\
= & \frac{1}{\Gamma(\gamma) \Gamma(1-s)} \times H_{1, m+1}^{2,1}\left[-\omega t^{\alpha} \mid \begin{array}{c}
(0,1) \\
(0,1),\left(1-s-\beta_{1}, \alpha_{1}\right),\left(1-s-\beta_{j}, \alpha_{j}\right)_{2, m}
\end{array}\right] \\
& \times M\left[t^{\beta_{1}} \varphi(t)\right] .
\end{aligned}
$$

\section{Laplace transform of the operator defined by (22)}

Theorem 3.1 It will be shown here that

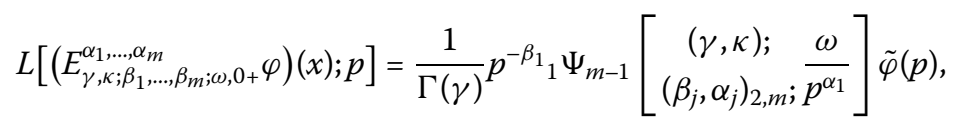

where $\alpha_{j}, \beta_{j}, \gamma, \kappa, \omega \in C, \operatorname{Re}\left(\alpha_{j}\right)>0, \operatorname{Re}\left(\beta_{j}\right)>0(j=1, \ldots, m) ; \operatorname{Re}\left(\sum_{j=1}^{m} \alpha_{j}\right)>\max [0, \operatorname{Re}(\kappa)-1]$, ${ }_{1} \Psi_{m-1}$ is the Fox-Wright function defined by

$$
L\left[\left(E_{\gamma ; \beta ; \omega, 0+}^{\alpha} \varphi\right)(x) ; p\right]=\frac{1}{\Gamma(\gamma)} p^{-\beta}\left[1-\frac{\omega}{p^{\alpha}}\right]^{-\gamma} \tilde{\varphi}(p),
$$

where $\alpha, \beta, \gamma, \omega \in C ; \operatorname{Re}(p)>0, \operatorname{Re}(\alpha)>0, \operatorname{Re}(\beta)>0, \operatorname{Re}(\gamma)>0$ and

$$
L[f(t) ; p]=\int_{0}^{\infty} e^{-p t} f(t) d t
$$

where $\operatorname{Re}(p)>0$ and the integral is convergent. 
Proof By virtue of the results (22) and (40), we find that

$$
\begin{aligned}
L & {\left[\left(E_{\gamma, \kappa ; \beta_{1}, \ldots, \beta_{m} ; \omega, 0+}^{\alpha_{1}, \ldots, \alpha_{m}} \varphi\right)(x) ; p\right] } \\
& =\int_{0}^{\infty} e^{-p x} \int_{0}^{x}(x-t)^{\beta_{1}-1} E_{\gamma, \kappa ; \beta_{1}, \ldots, \beta_{m}}^{\alpha_{1}, \ldots, \alpha_{m}}\left[\omega(x-t)^{\alpha_{1}}\right] \varphi(t) d t d x .
\end{aligned}
$$

Interchanging the order of integration, which is permissible under the conditions stated in Theorem 3.1, we find that

$$
\begin{aligned}
L & {\left[\left(E_{\gamma, \kappa ; \beta_{1}, \ldots, \beta_{m} ; \omega, 0+}^{\alpha_{1}, \ldots, \alpha_{m}} \varphi\right)(x) ; p\right] } \\
& =\int_{0}^{\infty} \varphi(t) \int_{t}^{\infty} e^{-p x}(x-t)^{\beta_{1}-1} E_{\gamma, \kappa ; \beta_{1}, \ldots, \beta_{m}}^{\alpha_{1}, \ldots, \alpha_{m}}\left[\omega(x-t)^{\alpha_{1}}\right] d x d t .
\end{aligned}
$$

If we set $x=t+u$ on the right-hand side of (41), it becomes

$$
\begin{aligned}
L & {\left[\left(E_{\gamma, \kappa ; \beta_{1}, \ldots, \beta_{m} ; \omega, a+}^{\alpha_{1}, \ldots, \alpha_{m}} \varphi\right)(x) ; p\right] } \\
& =\int_{0}^{\infty} \varphi(t) \int_{0}^{\infty} e^{-p(t+u)} u^{\beta_{1}-1} E_{\gamma, \kappa ; \beta_{1}, \ldots, \beta_{m}}^{\alpha_{1}, \ldots, \alpha_{m}}\left[\omega u^{\alpha_{1}}\right] d u d t .
\end{aligned}
$$

On making use of the series definition of function (10), we see that

$$
\begin{aligned}
L[ & \left.\left(E_{\gamma, \kappa ; \beta_{1}, \ldots, \beta_{m} ; \omega, 0+}^{\alpha_{1}, \ldots, \alpha_{m}} \varphi\right)(x) ; p\right] \\
& =\sum_{n=0}^{\infty} \frac{(\gamma)_{\kappa n} \omega^{n}}{\prod_{j=1}^{m} \Gamma\left(n \alpha_{j}+\beta_{j}\right)} \int_{0}^{\infty} e^{-p t} \varphi(t) d t \int_{0}^{\infty} e^{-p u} u^{\beta_{1}+\alpha_{1} n-1} d u \\
& =\sum_{n=0}^{\infty} \frac{(\gamma)_{\kappa n} \omega^{n}}{p^{\beta_{1}+\alpha_{1} n} \prod_{j=2}^{m} \Gamma\left(n \alpha_{j}+\beta_{j}\right)} \int_{0}^{\infty} e^{-p t} \varphi(t) d t \\
& =\frac{1}{\Gamma(\gamma)} p^{-\beta_{1}} \Psi_{m-1}\left[\begin{array}{c}
(\gamma, \kappa) ; \\
\left(\beta_{j}, \alpha_{j}\right)_{2, m} ;
\end{array} \frac{\omega}{p^{\alpha_{1}}}\right] \tilde{\varphi}(p),
\end{aligned}
$$

where $\tilde{\varphi}(p)$ is the Laplace transform of $\varphi(t)$.

This completes the proof of Theorem 3.1.

If we set $\kappa=q$, we obtain the following Corollary 3.1 .

Corollary 3.1 The following result holds:

$$
L\left[\left(E_{\gamma, q ; \beta_{1}, \ldots, \beta_{m} ; \omega, 0+}^{\alpha_{1}, \ldots, \alpha_{m}} \varphi\right)(x) ; p\right]=\frac{1}{\Gamma(\gamma)} p^{-\beta_{1}} \Psi_{m-1}\left[(\gamma, q) ;\left(\beta_{j}, \alpha_{j}\right)_{2, m} ; \frac{\omega}{p^{\alpha_{1}}}\right] \tilde{\varphi}(p)
$$

where $\alpha_{j}, \beta_{j}, \gamma, \omega \in C, \operatorname{Re}\left(\alpha_{j}\right)>0, \operatorname{Re}\left(\beta_{j}\right)>0(j=1, \ldots, m) ; \operatorname{Re}\left(\sum_{j=1}^{m} \alpha_{j}\right)>\max [0, q-1]$, $\operatorname{Re}(p)>0, \operatorname{Re}(\gamma)>0$.

When $m=1$, the above result reduces to the following.

Corollary 3.2 The following result holds:

$$
L\left[\left(E_{\gamma, q ; \beta ; \omega, 0+}^{\alpha} \varphi\right)(x) ; p\right]=\frac{1}{\Gamma(\gamma)} p_{1}^{-\beta} \Psi_{m-1}\left[(\gamma, q) ;-\frac{\omega}{p^{\alpha}}\right] \tilde{\varphi}(p)
$$


where $\alpha, \beta, \gamma, \omega \in C ; \operatorname{Re}(p)>0, \operatorname{Re}(\alpha)>0, \operatorname{Re}(\beta)>0, \operatorname{Re}(\gamma)>0,(q \in(0,1) \cup \mathrm{N})$, here $\tilde{\varphi}(p)$ is the Laplace transform of $\varphi(t)$.

When $q=1$, the above corollary yields the following result given in [6].

Corollary 3.3 There holds the formula

$$
L\left[\left(E_{\gamma ; \beta ; \omega, 0+}^{\alpha} \varphi\right)(x) ; p\right]=\frac{1}{\Gamma(\gamma)} p^{-\beta}\left[1-\frac{\omega}{p^{\alpha}}\right]^{-\gamma} \tilde{\varphi}(p),
$$

where $\alpha, \beta, \gamma, \omega \in C ; \operatorname{Re}(p)>0, \operatorname{Re}(\alpha)>0, \operatorname{Re}(\beta)>0, \operatorname{Re}(\gamma)>0$.

For $\gamma=\kappa=1$, we obtain the following result associated with the multiindex MittagLeffler function [16].

Corollary 3.4 There holds the formula

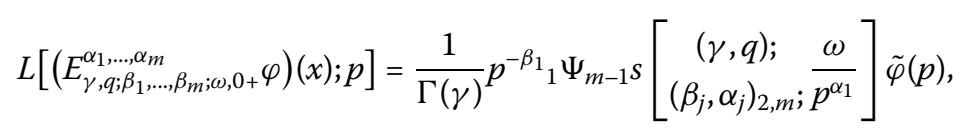

where $\alpha_{j}, \beta_{j}, \gamma, \omega \in C, \operatorname{Re}\left(\alpha_{j}\right)>0, \operatorname{Re}\left(\beta_{j}\right)>0(j=1, \ldots, m), \operatorname{Re}(p)>0, \operatorname{Re}(\gamma)>0$.

For $m=1$, Theorem 3.1 yields the following result [6].

Corollary 3.5 There holds the formula

$$
L\left[\left(E_{\gamma, \kappa ; \beta ; \omega, 0+}^{\alpha} \varphi\right)(x) ; p\right]=\frac{1}{\Gamma(\gamma)} p^{-\beta}{ }_{1} \Psi_{m-1}\left[\begin{array}{c}
(\gamma, \kappa) ; \\
-
\end{array} \frac{\omega}{p^{\alpha}}\right] \tilde{\varphi}(p),
$$

where $\alpha, \beta, \gamma, \omega \in C ; \operatorname{Re}(p)>0, \operatorname{Re}(\alpha)>\max [0, \operatorname{Re}(\kappa)-1], \operatorname{Re}(\beta)>0, \operatorname{Re}(\gamma)>0$, when $\gamma$ tends to zero and $\beta$ is replaced by $\alpha$, then by virtue of the limit formula (24), the result (10) reduces to the Riemann-Liouville fractional integral and we arrive at an important result given in Samko et al. [17].

Corollary 3.6 There holds the following result:

$$
L\left[\left(I_{0+}^{\alpha} \varphi\right)(x) ; p\right]=p^{-\alpha} \tilde{\varphi}(p)
$$

where $\alpha \in C ; \operatorname{Re}(p)>0, \operatorname{Re}(\alpha)>0$ and $\tilde{\varphi}(p)$ is the Laplace transform of $\varphi(t)$.

\section{Further properties of the operator defined by (22)}

By using the technique analogous to that employed by Kilbas et al. [29], we obtain the composition properties of the Riemann-Liouville fractional integral operator $I_{a+}^{\mu}$ involving the operator defined by (22).

Theorem 4.1 Under the various constraints already stated with definition (22), let the function $f$ be the nth space $L(a, b)$ of the Lebesgue measurable functions on finite interval $[a, b](b>a)$ of the real line $R$ given by

$$
L(a, b)=\left\{g(x):\left\|g_{1}\right\|_{1}\right\}=\int_{a}^{b}|g(x)| d x<\infty .
$$


The space $C(a, b)$ is of continuous functions on $[a, b]$, the integral defined by (22) is bounded on $L(a, b)$ and

$$
\left\|E_{\gamma, q ; \beta_{1}, \ldots, \beta_{m} ; \omega, 0+}^{\alpha_{1}, \ldots, \alpha_{m}} \varphi\right\| \leq d\|\varphi\|_{1}
$$

where the constant d is given by

$$
d=(b-a)^{\operatorname{Re}\left(\alpha_{1}\right)} \sum_{n=0}^{\infty} \frac{\left|(\gamma)_{\kappa n}\right|\left|\omega(b-a)^{\operatorname{Re}\left(\alpha_{1}\right)}\right|^{n}}{\prod_{j=1}^{m} \Gamma\left(n \alpha_{j}+\beta_{j}\right)\left|\operatorname{Re}\left(\alpha_{1}\right) n+\beta_{1}\right|(n) !} .
$$

For $\kappa=m=1$, (47) reduces to the one given by Kilbas et al. [29].

Theorem 4.2 Under the various constraints mentioned already with the parameters, there hold the following results:

$$
I_{a+}^{\alpha} E_{\gamma, \kappa ; \beta_{1}, \ldots, \beta_{m} ; \omega, 0+}^{\alpha_{1}, \ldots, \alpha_{m}} \varphi=E_{\gamma, \kappa ; \beta_{1}+\alpha, \ldots, \beta_{m} ; \omega, 0+}^{\alpha_{1}, \ldots, \alpha_{m}} \varphi=E_{\gamma, \kappa ; \beta_{1}, \ldots, \beta_{m} ; \omega, 0+}^{\alpha_{1}, \ldots, \alpha_{m}} I_{a+}^{\alpha} \varphi
$$

and

$$
D_{a+}^{\alpha} E_{\gamma, \kappa ; \beta_{1}, \ldots, \beta_{m} ; \omega, 0+}^{\alpha_{1}, \ldots, \alpha_{m}} \varphi=E_{\gamma, \kappa ; \beta_{1}-\alpha, \ldots, \beta_{m} ; \omega, 0+}^{\alpha_{1}, \ldots, \alpha_{m}} \varphi=E_{\gamma, \kappa ; \beta_{1}, \ldots, \beta_{m} ; \omega, 0+}^{\alpha_{1}, \ldots, \alpha_{m}} D_{a+}^{\alpha} \varphi .
$$

For $m=1,(48)$ and (49) reduce to the result given by Srivastava and Tomovski [11].

\section{Applications}

In this section, we present the solution of the following differential equation associated with a Hilfer derivative. The following result readily follows from paper [31]:

$$
L\left[E_{\gamma, \kappa}\left[\left(\alpha_{1}, \beta_{1}\right), \ldots,\left(\alpha_{m}, \beta_{m}\right) ; z\right] ; p\right]=\frac{1}{\Gamma(\gamma)} p^{-\beta_{1}}{ }_{1} \Psi_{m-1}\left[\begin{array}{c}
(\gamma, \kappa) ; \\
\left(\beta_{j}, \alpha_{j}\right)_{2, m} ;
\end{array} \frac{\omega}{p^{\alpha_{1}}}\right],
$$

where $\alpha_{j}, \beta_{j}, \gamma, \kappa \in C, \operatorname{Re}\left(\alpha_{j}\right)>0, \operatorname{Re}\left(\beta_{j}\right)>0(j=1, \ldots, m) ; \operatorname{Re}(\kappa)>0, \operatorname{Re}\left(\sum_{j=1}^{m} \alpha_{j}\right)>$ $\max [0, \kappa-1]$ and $\operatorname{Re}(p)>0$.

Theorem 5.1 It will be shown here that the solution of the fractional differential equation

$$
\left(D_{0+}^{\mu, v} f\right)(x)=\lambda\left(E_{\gamma, \kappa ; \beta_{1}, \ldots, \beta_{m} ; \omega, a+}^{\alpha_{1}, \ldots, \alpha_{m}} \omega x^{\alpha_{1}}\right)(x)+g(x)
$$

where $0<\mu<1,0 \leq v \leq 1, \omega \in C, \operatorname{Re}\left(\sum_{j=1}^{m} \alpha_{j}\right)>\max [0, \operatorname{Re}(\kappa)-1], \operatorname{Re}\left(\beta_{j}\right)>0(j=1, \ldots, m)$, $\min \{\operatorname{Re}(\gamma), \operatorname{Re}(\kappa)\}>0$,

$$
\left(I_{0+}^{(1-v)(1-\mu)} f\right)(0+)=e,
$$

has the solution given by

$$
\begin{aligned}
f(x)= & e \frac{x^{\mu-\nu(\mu-1)-1}}{\Gamma(\mu-v+\mu \nu)}+\frac{\lambda x^{\mu+\beta_{1}}}{\Gamma(\gamma)} E_{\gamma, \kappa}\left[\left(\alpha_{1}, \beta_{1}+\mu+1\right), \ldots,\left(\alpha_{m}, \beta_{m}\right) ; \omega x^{\alpha_{1}}\right] \\
& +\frac{1}{\Gamma(\mu)} \int_{0}^{x}(x-t)^{\mu-1} g(t) d t
\end{aligned}
$$

where e is an arbitrary constant. 
Proof Applying the Laplace transform to each side of (53) and using (21) along with convolution theorem of the Laplace transform, we find that

$$
\begin{aligned}
& p^{\mu} F(p)-e p^{\nu(1-\mu)}=L\left[\left(E_{\gamma, \kappa ; \beta_{1}, \ldots, \beta_{m} ; \omega, a+}^{\alpha_{1}, \ldots, \alpha_{m}} \varphi\right) ; p\right] L(1 ; p)+G(p)
\end{aligned}
$$

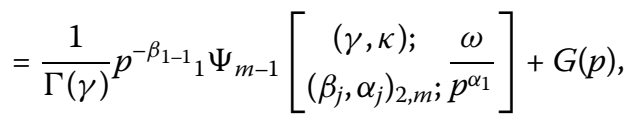

which readily gives

$$
F(p)=e p^{\nu(1-\mu)-\mu}+\frac{\lambda}{\Gamma(\gamma)} p^{-\beta_{1}-\mu-1} \sum_{n=0}^{\infty} \frac{\Gamma(\gamma+\kappa n)\left(\frac{\omega}{p^{\alpha_{1}}}\right)^{n}}{\prod_{j=1}^{m} \Gamma\left(n \operatorname{Re}\left(\alpha_{j}\right)+\beta_{j}\right)(n !)}+p^{-\mu} G(p) .
$$

Now, by taking the inverse Laplace transform of each side of (55), we obtain

$$
\begin{aligned}
F(x)= & e L^{-1}\left(p^{\nu(1-\mu)-\mu} ; x\right)+\frac{\lambda}{\Gamma(\gamma)} \sum_{n=0}^{\infty} \frac{\Gamma(\gamma+\kappa n)\left(\frac{\omega}{p^{\alpha_{1}}}\right)^{n}}{\prod_{j=1}^{m} \Gamma\left(n \operatorname{Re}\left(\alpha_{j}+\beta_{j}\right)\right)(n !)} L^{-1}\left(p^{-\alpha_{1} n-\beta_{1}-\mu-1} ; x\right) \\
& +L^{-1} L\left(\frac{x^{\mu-1}}{\Gamma(\mu)} * g(x)\right) \\
= & e \frac{x^{\mu-\nu(1-\mu)-1}}{\Gamma(\mu-v(1-\mu))}+\frac{\lambda x^{\mu+\beta_{1}}}{\Gamma(\gamma)} \sum_{n=0}^{\infty} \frac{\Gamma(\gamma+\kappa n)\left(\omega x^{\alpha_{1}}\right)^{n}}{\prod_{j=1}^{m} \Gamma\left(n \operatorname{Re}\left(\alpha_{j}\right)+\beta_{j}\right) \Gamma\left(\alpha_{1} n+\beta_{1}+\mu+1\right)(n) !} \\
& +\frac{1}{\Gamma(\mu)} \int_{0}^{x}(x-t)^{\mu-1} g(t) d t,
\end{aligned}
$$

which yields

$$
\begin{aligned}
f(x)= & e \frac{x^{\mu-v(1-\mu)-1}}{\Gamma(\mu-v(1-\mu))}+\frac{\lambda x^{\mu+\beta_{1}}}{\Gamma(\gamma)} E_{\gamma, \kappa}\left[\left(\alpha_{1}, \beta_{1}+\mu+1\right), \ldots,\left(\alpha_{m}, \beta_{m}\right) ; \omega x^{\alpha_{1}}\right] \\
& +\frac{1}{\Gamma(\mu)} \int_{0}^{x}(x-t)^{\mu-1} g(t) d t .
\end{aligned}
$$

This completes the proof of Theorem 5.1.

It is interesting to observe that for $m=1$, Theorem 5.1 reduces to the one given by Srivastava and Tomovski [11].

\section{Conclusion}

In this paper we have derived the Laplace transform and the Mellin transform and various properties of the operator defined by (21). These properties are useful in solving applied problems of science, engineering and technology such as fractional kinetic equations, fractional reaction-diffusion equation, etc.

\section{Authors' contributions}

The authors contributed equally and significantly in writing this article under the supervision of Prof. R. K. Saxena. The authors read and approved the final manuscript. 


\section{Author details}

'Department of Mathematics and Statistics, Jai Narain Vyas University, Jodhpur, 342004, India. ${ }^{2}$ Department of Applied Mathematics \& Humanities, S.V. National Institute of Technology, Surat, 395007, India.

\section{Received: 20 November 2014 Accepted: 4 February 2015 Published online: 01 March 2015}

\section{References}

1. Mittag-Leffler, GM: Sur la nouvelle fonction $E_{\alpha}(x)$. C. R. Acad. Sci. Paris 137, 554-558 (1903)

2. Mittag-Leffler, GM: Sur la représentation analytique d'une fonction monogène (cinquieme note). Acta Math. 29 101-181 (1905)

3. Wiman, A: Über den Fundamentalsatz in der Theorie der Funktionen $E_{\alpha}(x)$. Acta Math. 29, 191-201 (1905)

4. Prabhakar, TR: A singular integral equation associated with a generalized Mittag-Leffler function in the kernel. Yokohama Math. J. 19, 7-15 (1971)

5. Shukla, AK, Prajapati, JC: On generalization of Mittag-Leffler function and its properties. J. Math. Anal. Appl. 336, 797-811 (2007)

6. Prajapati, JC, Jana, RK, Saxena, RK, Shukla, AK: Some results on generalized Mittag-Leffler function operator. J. Inequal. Appl. 2013, 33 (2013)

7. Wright, EM: The asymptotic expansion of generalized hypergeometric function. J. Lond. Math. Soc. 10, 286-293 (1935)

8. Wright, EM: The asymptotic expansion of generalized hypergeometric function. Proc. Lond. Math. Soc. 27, 389-408 (1940)

9. Mainardi, F, Pagnini, G: The Wright function as solution of time-fractional diffusion equation. Appl. Math. Comput. $141,51-62(2003)$

10. Kilbas, AA, Saigo, HM, Trujillo, JJ: On the generalized Wright function. Fract. Calc. Appl. Anal. 4, 437-460 (2002)

11. Srivastava, HM, Tomovski, Z: Fractional calculus with an integral operator containing a generalized Mittag-Leffler function in the kernel. Appl. Math. Comput. 211, 198-210 (2009)

12. Saxena, RK, Ram, J, Vishnoi, M: Fractional differentiation and fractional integration of the generalized Mittag-Leffler function. J. Indian Acad. Math. 32(1), 153-162 (2010)

13. Saxena, RK, Nishimoto, K: Further results on the generalized Mittag-Leffler functions of fractional calculus. J. Fract. Calc. 40, 29-41 (2011)

14. Saxena, RK, Nishimoto, K: N-Fractional calculus of generalized Mittag-Leffler functions. J. Fract. Calc. 37, 43-52 (2010)

15. Kiryakova, V: Multiple (multiindex) Mittag-Leffler function related Gelfond and Leontiex operators and Laplace type integrals transforms. Fract. Calc. Appl. Anal. 2, 445-462 (1999)

16. Kiryakova, V: Multiple (multiindex) Mittag-Leffler functions and relations to generalized fractional calculus. J. Comput. Appl. Math. 118, 214-259 (2000)

17. Saxena, RK, Kalla, SL, Kiryakova, VS: Relations connecting multiindex Mittag-Leffler functions and Riemann-Liouville fractional calculus. Algebras Groups Geom. 20, 365-385 (2003)

18. Hilfer, R: Fractional time evolution. In: Applications of Fractional Calculus in Physics. World Scientific, Singapore (2000)

19. Kilbas, AA, Srivastava, HM, Trujillo, JJ: Theory and Applications of Fractional Differential Equations. Elsevier, Amsterdam (2006)

20. Mathai, AM, Saxena, RK, Haubold, HJ: The H-Function: Theory and Applications. Springer, New York (2010)

21. Samko, SG, Kilbas, AA, Marichev, Ol: Fractional Integrals and Derivatives: Theory and Applications. Gordon \& Breach, New York (1993)

22. Srivastava, HM, Saxena, RK: Operators of fractional integration and their applications. Appl. Math. Comput. 118, 1-52 (2001)

23. Erdélyi, A, Magnus, W, Oberhettinger, F, Tricomi, FG: Tables of Integral Transforms, vol. 2. McGraw-Hill, New York (1954)

24. Caputo, M: Elasticità e Dissipazione. Zanichelli, Bologna (1969)

25. Prudnikov, AP, Brychkov, YA, Marichev, Ol: Integrals and Series: More Special Functions, vol. 3. Gordon \& Breach, New York (1990)

26. Gorenflo, R, Luchko, Y, Mainardi, F: Analytic properties and applications of the Wright function. Fract. Calc. Appl. Anal. 2, 383-414 (1999)

27. Górska, K, Penson, KA, Babusci, D, Dattoli, G, Duchamp, GHE: Operator solutions for fractional Fokker-Planck equations. Phys. Rev. E 85, 031138 (2012)

28. Babusci, D, Dattoli, G, Górska, K: On Mittag-Leffler function and associated polynomials (2012). arXiv:1206.3495

29. Kilbas, AA, Saigo, M, Saxena, RK: Generalized Mittag-Leffler function and generalized fractional calculus. Integral Transforms Spec. Funct. 15, 31-49 (2004)

30. Erdélyi, A, Magnus, W, Oberhettinger, F, Tricomi, FG: Higher Transcendental Functions, vol. 3. McGraw-Hill, New York (1955)

31. Srivastava, HM, Saxena, RK, Ram, C: A unified presentation of gamma-type functions occurring in diffraction theory and associated probability distributions. Appl. Math. Comput. 162, 931-947 (2005) 